\title{
Isolation and cross-species characterization of polymorphic microsatellites for the orchid bee Eulaema meriana (Hymenoptera: Apidae: Euglossini)
}

\author{
Margarita M. López-Uribe • Amy N. Green • \\ Santiago R. Ramírez · Steve M. Bogdanowicz • \\ Bryan N. Danforth
}

Received: 1 April 2010/Accepted: 16 April 2010/Published online: 9 July 2010

(C) The Author(s) 2010. This article is published with open access at Springerlink.com

\begin{abstract}
We describe and characterize eight polymorphic microsatellite loci for the orchid bee species Eulaema meriana, an abundant species and important pollinator in wet lowland forests in tropical America. We also tested the cross-species amplification of these microsatellite loci in seven other species of the genus Eulaema. For E. meriana, number of alleles per locus ranged from four to nine and expected heterozygosity ranged from 0.377 to 0.854 . Seven out of the eight loci described amplified in all seven other Eulaema species. These microsatellite loci will be of practical use for population structure, mating system and inbreeding studies in euglossine bees.
\end{abstract}

Keywords Eulaema $\cdot$ Euglossine bees $\cdot$ Microsatellites . Diploid males

The tribe Euglossini comprises 218 species in 5 genera, including the genus Eulaema with 29 described species (Oliveira 2006; Nemésio 2009). Euglossine bees (Hymenoptera: Apidae), commonly known as orchid bees, are charismatic insects characterized by extremely long tongues and shiny iridescent colors (Roubik and Hanson

M. M. López-Uribe $(\bowtie) \cdot$ A. N. Green · B. N. Danforth Department of Entomology, Cornell University, Ithaca, NY 14853, USA

e-mail:mm182@cornell.edu

\section{S. R. Ramírez}

Department of Environmental Sciences, University of California Berkeley, Berkeley, CA 94720, USA

\section{S. M. Bogdanowicz}

Evolutionary Genetics Core Facilities, Department of Ecology and Evolutionary Biology, Cornell University, Ithaca,

NY 14853, USA
2004). Orchid bees are abundant in the Neotropics (LópezUribe et al. 2008) and are considered keystone species in lowland forests because of the ecological role that they play as pollinator of orchids (Dressler 1982) and many other flowering plants (Ramírez et al. 2002).

Orchid bees have recently been targets of conservation concern (Zayed 2004). There is evidence demonstrating that the species diversity of euglossine bees is negatively affected by habitat fragmentation (Brosi 2009). In addition, genetic studies using allozyme markers have shown that some orchid bee populations exhibit high frequencies of diploid males indicating high levels of inbreeding and/or low effective population size (Roubik et al. 1996; Zayed 2004; López-Uribe et al. 2007; but see Takahashi et al. 2001). However, a recent study looking for diploid males using microsatellite markers (Souza et al. 2010) found diploid males to be rare in euglossine bee populations suggesting that the high frequency of diploid males previously reported may be the result of technical flaws in the allozyme-based studies. Therefore, the development of microsatellite markers is essential for the study of population structure and conservation genetics of this group of bees. Here, we describe and characterize eight polymorphic microsatellite loci in Eulaema meriana, and tested these loci across seven other Eulaema species.

A genomic DNA library enriched for 12 microsatellite repeat motifs was created from one individual of E. meriana using a universal linker and ligation procedure (Hamilton et al. 1999; Grant and Bogdanowicz 2006). Transformed bacterial colonies were then screened for microsatellites through hybridization to ${ }^{33} \mathrm{P}$-radiolabeled oligonucleotides. More than 800 positive clones were obtained from this method and $\sim 200$ of them were sequenced with universal M13 primers that flank the cloned insert for microsatellite primer design. PCR primer 
Table 1 Primer sequences, repeat motif and annealing temperature $\left(T_{\mathrm{a}}\right)$ of eight microsatellite loci isolated from Eulaema meriana

\begin{tabular}{|c|c|c|c|c|c|c|c|}
\hline Locus & Primer sequence $\left(5^{\prime} \rightarrow 3^{\prime}\right)$ & Repeat motif & $T_{\mathrm{a}}$ & $\begin{array}{l}\text { Allele } \\
\text { size (bp) }\end{array}$ & $N_{\mathrm{A}}$ & $H_{\mathrm{E}}$ & $\begin{array}{l}\text { GenBank } \\
\text { accession no }\end{array}$ \\
\hline EM8 & $\begin{array}{l}\text { F: CAG CGT CGC GAT TGG TTC TAC A } \\
\text { R: TCA GCT TTG TCA CCG GCA CTG T }\end{array}$ & $(\mathrm{GA})_{14}$ & 55 & $301-317$ & 6 & 0.723 & GU997087 \\
\hline EM13 & $\begin{array}{l}\text { F: GGC GCA ATG ACT AAG GGA ACG } \\
\text { R: CCC ACG GGC TAA CGA TGT ATC TT }\end{array}$ & $(\mathrm{TGC})_{7}$ & 55 & $173-185$ & 4 & 0.637 & GU997088 \\
\hline EM16 & $\begin{array}{l}\text { F: AGC GCA ATT ACA TAT GCA AAA ACA } \\
\text { R: TCC GGT GGT ATC TGA GCA TTA TTC }\end{array}$ & $(\mathrm{CAG})_{6}(\mathrm{CCACAG})_{2}$ & 55 & $190-211$ & 4 & 0.377 & GU997089 \\
\hline EM17 & $\begin{array}{l}\text { F: GGG CGA CGG CGA AGA TTT } \\
\text { R: CGT TGC GCC CGA CTT TAC A }\end{array}$ & $(\mathrm{CTT})_{10}$ & 55 & $157-187$ & 6 & 0.731 & GU997090 \\
\hline EM40 & $\begin{array}{l}\text { F: CGA CGC AGA CGC AGC AAC AG } \\
\text { R: CCC GCG GAC TAA ACG ACA ACA CT }\end{array}$ & $(\mathrm{CAA})_{10}$ & 57 & $143-164$ & 7 & 0.722 & GU997091 \\
\hline EM70 & $\begin{array}{l}\text { F: GTA CCA CTG CGA GAG CGA AGA AAA } \\
\text { R: CCA GTG GCC CGA AGT AGA AAC A }\end{array}$ & $(\mathrm{AG})_{2} \mathrm{G}(\mathrm{AG})_{9} \mathrm{~A}(\mathrm{AG})_{7} \mathrm{~A}(\mathrm{AG})_{6}$ & 55 & $280-288$ & 5 & 0.596 & GU997092 \\
\hline EM106 & $\begin{array}{l}\text { F: GAC GTG GAT GAG CCG CAG AAG AC } \\
\text { R: TCC GAC GAT GTA CGA GCA CGA A }\end{array}$ & $(\mathrm{AAG})_{9} \mathrm{GAG}(\mathrm{AAG})_{9}$ & 55 & $261-300$ & 9 & 0.854 & GU997093 \\
\hline EM107 & $\begin{array}{l}\text { F: CGA GCC CCG ACG ACG AAC } \\
\text { R: GAC CGG AAC GAG CTG GAT GAA T }\end{array}$ & $(\mathrm{TCT})_{2} \mathrm{TCC}(\mathrm{TCT})_{12}$ & 57 & $200-221$ & 8 & 0.841 & GU997094 \\
\hline
\end{tabular}

Allele size range, number of alleles $\left(N_{\mathrm{A}}\right)$ and expected heterozygosity $\left(H_{\mathrm{E}}\right)$ were calculated for the population from La Selva, Costa Rica $(N=40$ haploid males $)$

Table 2 Characterization of microsatellite loci isolated from Eulaema meriana from four localities and cross-species amplification for seven other species of the genus Eulaema

\begin{tabular}{|c|c|c|c|c|c|c|c|c|}
\hline Locus & $\begin{array}{l}\text { Eulaema } \\
\text { meriana } \\
(N=55)\end{array}$ & $\begin{array}{l}\text { Eulaema } \\
\text { cingulata } \\
(N=15) \\
\end{array}$ & $\begin{array}{l}\text { Eulaema } \\
\text { bombiformis } \\
(N=10)\end{array}$ & $\begin{array}{l}\text { Eulaema } \\
\text { chocoana } \\
(N=3)\end{array}$ & $\begin{array}{l}\text { Eulaema } \\
\text { luteola } \\
(N=3)\end{array}$ & $\begin{array}{l}\text { Eulaema } \\
\text { mocsaryi } \\
(N=2)\end{array}$ & $\begin{array}{l}\text { Eulaema } \\
\text { nigrifacies } \\
(N=1)\end{array}$ & $\begin{array}{l}\text { Eulaema } \\
\text { nigrita } \\
(N=1)\end{array}$ \\
\hline EM8 & $\begin{array}{l}8 \\
(301-323)\end{array}$ & $\begin{array}{l}3 \\
(301-311)\end{array}$ & $\begin{array}{l}3 \\
(307-313)\end{array}$ & $\begin{array}{l}3 \\
(309-315)\end{array}$ & $\begin{array}{l}2 \\
(290-292)\end{array}$ & $\begin{array}{l}1 \\
(301)\end{array}$ & $\begin{array}{l}1 \\
(317)\end{array}$ & $\begin{array}{l}1 \\
(309)\end{array}$ \\
\hline EM13 & $\begin{array}{l}4 \\
(173-185)\end{array}$ & $\begin{array}{l}6 \\
(161-185)\end{array}$ & $\begin{array}{l}5 \\
(173-188)\end{array}$ & $\begin{array}{l}2 \\
(197-203)\end{array}$ & $\begin{array}{l}2 \\
(192-198)\end{array}$ & $\begin{array}{l}1 \\
(173)\end{array}$ & $\begin{array}{l}1 \\
(164)\end{array}$ & $\begin{array}{l}1 \\
(182)\end{array}$ \\
\hline EM16 & $\begin{array}{l}4 \\
(190-211)\end{array}$ & $\begin{array}{l}2 \\
(199-202)\end{array}$ & $\begin{array}{l}5 \\
(201-216)\end{array}$ & $\begin{array}{l}1 \\
(202)\end{array}$ & $\begin{array}{l}1 \\
(202)\end{array}$ & $\begin{array}{l}1 \\
(199)\end{array}$ & $\begin{array}{l}1 \\
(210)\end{array}$ & $\begin{array}{l}1 \\
(190)\end{array}$ \\
\hline EM17 & $\begin{array}{l}7 \\
(157-187)\end{array}$ & $\begin{array}{l}2 \\
(169-184)\end{array}$ & $\begin{array}{l}5 \\
(178-196)\end{array}$ & $\begin{array}{l}1 \\
(172)\end{array}$ & $\begin{array}{l}1 \\
(157)\end{array}$ & $\begin{array}{l}1 \\
(184)\end{array}$ & $\begin{array}{l}1 \\
(178)\end{array}$ & $\begin{array}{l}1 \\
(220)\end{array}$ \\
\hline EM40 & $\begin{array}{l}9 \\
(140-167)\end{array}$ & $\begin{array}{l}4 \\
(143-158)\end{array}$ & $\begin{array}{l}5 \\
(152-173)\end{array}$ & $\begin{array}{l}1 \\
(147)\end{array}$ & $\begin{array}{l}2 \\
(158-164)\end{array}$ & $\begin{array}{l}2 \\
(161-164)\end{array}$ & $\begin{array}{l}1 \\
(164)\end{array}$ & $\begin{array}{l}1 \\
(167)\end{array}$ \\
\hline EM70 & $\begin{array}{l}5 \\
(280-288)\end{array}$ & $\begin{array}{l}5 \\
(284-294)\end{array}$ & $\begin{array}{l}2 \\
(261-294)\end{array}$ & $\begin{array}{l}1 \\
(288)\end{array}$ & $\begin{array}{l}3 \\
(225-273)\end{array}$ & $\begin{array}{l}1 \\
(287)\end{array}$ & $\begin{array}{l}1 \\
(297)\end{array}$ & $\begin{array}{l}1 \\
(297)\end{array}$ \\
\hline EM106 & $\begin{array}{l}11 \\
(261-300)\end{array}$ & $\begin{array}{l}5 \\
(268-295)\end{array}$ & $\begin{array}{l}7 \\
(261-294)\end{array}$ & $\begin{array}{l}1 \\
(264)\end{array}$ & $\begin{array}{l}2 \\
(225-273)\end{array}$ & $\begin{array}{l}2 \\
(271-283)\end{array}$ & $\begin{array}{l}1 \\
(282)\end{array}$ & $\begin{array}{l}1 \\
(305)\end{array}$ \\
\hline EM107 & $\begin{array}{l}8 \\
(200-221)\end{array}$ & $\begin{array}{l}4 \\
(196-221)\end{array}$ & $\begin{array}{l}3 \\
(203-212)\end{array}$ & $\begin{array}{l}1 \\
(203)\end{array}$ & $\begin{array}{l}1 \\
(199)\end{array}$ & $\begin{array}{l}2 \\
(199-202)\end{array}$ & $\begin{array}{l}1 \\
(202)\end{array}$ & - \\
\hline
\end{tabular}

Above: number of alleles per locus; below: allele range size per locus $(N=$ number of haploid males)

pairs were designed for 29 microsatellite loci using the software PrimerSelect (DNASTAR). Nine of these loci were tested for PCR amplification quality and variability.

For microsatellite PCR amplifications, a universal tag method with three primers was employed (Schuelke 2000).
This approach allows fluorescent labeling of PCR fragments with a single dye-labeled tag used simultaneously with the unlabeled locus-specific (ULS) forward primer containing 20 additional bases at the $5^{\prime}$-end and the ULS reverse primer. The reverse primer was modified by adding 
a six base pair 'pigtail' (GTTTCT) to the 5'-end (Brownstein et al. 1996) to facilitate genotyping by reducing stutter. PCR amplifications contained $5 \times$ GoTaq buffer $\mathrm{pH}$ $8.5,2 \mathrm{mM} \mathrm{MgCl} 2,0.2 \mathrm{mM}$ dNTPs, $0.1 \mu \mathrm{M}$ ULS forward primer, $0.2 \mu \mathrm{M}$ dye-labeled tag, $0.2 \mu \mathrm{M}$ ULS reverse primer, 1U GoTaq DNA polymerase (Promega) and 10-50 ng DNA in $20 \mu \mathrm{l}$ total volume. PCR cycling conditions consisted of one cycle at $94^{\circ} \mathrm{C}$ for $30 \mathrm{~s}, 35$ cycles at $94^{\circ} \mathrm{C}$ for $30 \mathrm{~s}, 45 \mathrm{~s}$ at the locus-specific annealing temperature (Table 1) and $45 \mathrm{~s}$ at $72^{\circ} \mathrm{C}$, followed by one step of $7 \mathrm{~min}$ at $72^{\circ} \mathrm{C}$. Cycling was carried out using a Biometra TGradient thermal cycler. Labeled PCR products were analyzed on an Applied BioSystems 3730 1× DNA Analyzer using the allele size standard GeneScan-500 LIZ and called using the software PeakScanner (Applied BioSystems).

Genomic DNA was extracted from males of E. meriana $(N=55)$, Eulaema cingulata $(N=15)$, Eulaema bombiformis $(N=10)$, Eulaema chocoana $(N=3)$, Eulaema luteola $(N=2)$, Eulaema mocsaryi $(N=2)$, Eulaema nigrifacies $(N=1)$ and Eulaema nigrita $(N=1)$ (Table 1) using the QIAGEN DNeasy Tissue kit. Characterization of each locus was based on one E. meriana population $(N=40)$ from La Selva, Costa Rica (Table 1). All loci were checked for amplification variability in four E. meriana populations and across the other seven Eulaema species (Table 2). Due to the haploid nature of the data, tests for Hardy-Weinberg equilibrium and linkage disequilibrium were not performed. Number of alleles per locus $\left(N_{\mathrm{A}}\right)$ and expected heterozygosity $\left(H_{\mathrm{E}}\right)$ were calculated using Microsatellite Analyser (MSA) (Dieringer and Schlotterer 2003).

The number of alleles per locus for E. meriana ranged from 4 to 9 in the population from La Selva (Costa Rica) (Table 1) and from 4 to 11 when including individuals from the other 3 populations analyzed (Table 2). Null alleles were only detected for the locus EM40 in one $E$. meriana individual. All microsatellite loci were easily genotyped in all species except for locus EM107 in $E$. luteola and E. nigrita. Stutter was only evident in locus EM70 for E. luteola. None of the 90 individuals analyzed showed a diploid genotype. Successful cross-species amplification of these loci shows that the microsatellite markers here described will be useful tools for future population and conservation genetic studies in E. meriana and several species of the genus Eulaema.

Acknowledgments We would like to thank R. Paxton, A. Soro and S. Cardinal for their comments on the manuscript. This work was supported by the Griswold Endowment, the Organization of Tropical Studies and the Lewis and Clark Fund for Exploration.
Open Access This article is distributed under the terms of the Creative Commons Attribution Noncommercial License which permits any noncommercial use, distribution, and reproduction in any medium, provided the original author(s) and source are credited.

\section{References}

Brosi B (2009) The effects of forest fragmentation on euglossine bee communities (Hymenoptera: Apidae: Euglossini). Biol Conserv 142:414-423

Brownstein MJ, Carpten JD, Smith JR (1996) Modulation of nontemplated nucleotide addition by Taq DNA Polymerase: primer modifications that facilitate genotyping. BioTechniques 20:1004-1010

Dieringer D, Schlotterer C (2003) Microsatellite Analyser (MSA): a platform independent analysis tool for large microsatellite data sets. Mol Ecol Notes 3:167-169

Dressler RL (1982) Biology of the orchid bees (Euglossini). Annu Rev Ecol Syst 13:373-392

Grant J, Bogdanowicz S (2006) Isolation and characterization of microsatellite markers from the panic moth, Saucrobotys futilalis L. (Lepidoptera: Pyralidae: Pyraustinae). Mol Ecol Notes 6:353355

Hamilton MB, Pincus EL, Di Fiore A, Fleischer RC (1999) Universal linker and ligation procedures for construction of genomic DNA libraries enriched for microsatellites. BioTechniques 27:500-506

López-Uribe MM, Almanza MT, Ordóñez M (2007) Diploid male frequencies in Colombian populations of euglossine bees (Hymenoptera: Apidae: Euglossini). Biotropica 39:660-662

López-Uribe MM, Oi CA, Del Lama MA (2008) Nectar-foraging behavior of euglossine bees (Hymenoptera: Apidae) in urban areas. Apidologie 39:410-418

Nemésio A (2009) Orchid bees (Hymenoptera: Apidae) of the Brazilian Atlantic forest. Zootaxa 2041:242

Oliveira ML (2006) Nova hipótese de relacionamento filogenético entre os gêneros de Euglossini e entre as espécies de Eulaema Lepeletier, 1841 (Hymenoptera: Apidae: Euglossini). Acta Amazonica 36:273-286

Ramírez S, Dressler RL, Ospina M (2002) Abejas euglossinas (Hymenoptera: Apidae) de la región neotropical: listado de especies con notas sobre su biología. Biota Colombiana 3:7-118

Roubik DW, Hanson PE (2004) Orchid Bees of Tropical America: Biology and Field Guide. Instituto Nacional de Biodiversidade (INBio), Santo Domingo de Heredia

Roubik DW, Weight LA, Bonilla MA (1996) Population genetics, diploid males, and limits to social evolution of euglossine bees. Evolution 50:931-935

Schuelke M (2000) An economic method for the fluorescent labeling of PCR fragments. Nat Biotechnol 18:233-234

Souza RO, Del Lama MA, Cervini C, Mortari N, Eltz T, Zimmermann Y, Bach C, Brosi BJ, Suni S, Quezada-Euán JJG, and Paxton RJ (2010) Conservation genetics of Neotropical pollinators revisited: microsatellite analysis demonstrates that diploid males are rare in orchid bees. Evolution. doi:10.1111/j.1558-5646.2010.01052.x

Takahashi NC, Peruquetti RC, Del Lama MA, Campos LAO (2001) A reanalysis of diploid male frequencies in euglossine bees (Hymenoptera: Apidae). Evolution 55:1897-1899

Zayed A (2004) Effective population size in Hymenoptera with complementary sex determination. Heredity 93:627-630 